\title{
Influence of the Weibel instability on the expansion of a plasma slab into a vacuum
}

\author{
C. Thaury, P. Mora, A. Héron, and J. C. Adam \\ Centre de Physique Théorique, Ecole Polytechnique-CNRS, 91128 Palaiseau, France \\ T. M. Antonsen \\ Department of Electrical Engineering, University of Maryland, College Park, Maryland 20742, USA
}

(Received 18 June 2010; published 25 August 2010)

\begin{abstract}
The development of the Weibel instability during the expansion of a thin plasma foil heated by an intense laser pulse is investigated, using both analytical models and relativistic particle-in-cell simulations. When the plasma has initially an anisotropic electron distribution, this electromagnetic instability develops from the beginning of the expansion. Then it contributes to suppress the anisotropy and eventually saturates. After the saturation, the strength of the magnetic field decreases because of the plasma expansion until it becomes too weak to maintain the distribution isotropic. For this time, the anisotropy rises as electrons give progressively their longitudinal energy to ions, so that a new instability can develop.
\end{abstract}

DOI: 10.1103/PhysRevE.82.026408

PACS number(s): 52.38.Fz, 52.35.-g, 52.65.Rr, 52.38.Kd

\section{INTRODUCTION}

When an intense laser pulse impinges on a thin solid target, it ionizes it within a few optical cycles and forms a dense plasma with an electron temperature in the $0.1-1 \mathrm{keV}$ range. The laser pulse then interacts with the plasma around the critical density surface, where it is reflected, and heats a population of electrons, up to $\mathrm{MeV}$ energies for laser intensities $I \lambda^{2}>10^{19} \mathrm{~W} \mathrm{~cm}^{-2} \mu \mathrm{m}^{2}$ [1]. These hot electrons flow through the target, and a part of them forms electrostatic sheaths, on both sides of the plasma, which pull ions into the vacuum and accelerates them, potentially up to several tens of $\mathrm{MeV}$ [2-10].

This acceleration process is commonly described using one-dimensional (1D) models of plasma expansion into a vacuum [11-14]. The assumption of a dependence on a single spatial dimension is justified as long as the laser focal spot is sufficiently large. However, it is incorrect to consider only one component of the electron velocities. Indeed, several mechanisms such as elastic Coulomb collisions and electromagnetic instabilities can induce a coupling between the transverse and longitudinal components of velocity. These can strongly affect both the expansion dynamics $[15,16]$ and the properties of the accelerated ion beams $[17,18]$.

In this paper, we assume that the plasma is too hot for collisions to be efficient [15] and consider the influence of the Weibel instability on the expansion. The principle of this electromagnetic instability, which occurs in plasmas with anisotropic velocity distributions, is schematized in Fig. 1. We assume that $\left(\boldsymbol{e}_{x}, \boldsymbol{e}_{\boldsymbol{y}}, \boldsymbol{e}_{z}\right)$ is a standard basis, and that the initial electron velocity distribution function is $f^{0}(\boldsymbol{v})$ $\propto \exp \left[-v_{x}^{2} / v_{x 0}^{2}-v_{y}^{2} / v_{y 0}^{2}-v_{z}^{2} / v_{z 0}^{2}\right]$ with $\boldsymbol{v}=\left(v_{x}, v_{y}, v_{z}\right)$ and $v_{x 0}$ $>v_{y 0}=v_{z 0}$. We also suppose that a perturbation magnetic field $\boldsymbol{B}=B^{0} \cos k x \boldsymbol{e}_{z}$ arises from the noise. In these conditions, the magnetic Lorentz force bends the electron trajectories in the $\left(\boldsymbol{e}_{\boldsymbol{x}}, \boldsymbol{e}_{\boldsymbol{y}}\right)$ plane (see Fig. 1) and forms current sheets $\boldsymbol{j}=\boldsymbol{j} \boldsymbol{e}_{\boldsymbol{x}}$ which enhance the magnetic field [19]. As $\boldsymbol{B}$ does not perturb the isotropic part of $f^{0}$ the currents build always along a hot axis and the instability develops only in anisotropic plasmas.
Such plasmas are naturally obtained when thin foils are irradiated by intense laser pulses. Indeed hot electrons gain most of their energy through collisionless mechanisms (e.g., resonant absorption [20], Brunel absorption [21], and $\boldsymbol{j} \times \boldsymbol{B}$ heating [22]) which depend on the polarization of the laser and heat the plasma in a preferred direction. Because of this anisotropy which can be very strong, the Weibel instability is triggered from the beginning of the expansion (see the growth of the mean magnetic energy by hot electron in Fig. 2 ). As electrons give progressively their longitudinal momenta to ions through the ambipolar field, and transfer a part of it to the transverse directions through the magnetic Lorentz force, the anisotropy is eventually suppressed (at $t$ $\approx 6 \mathrm{fs}$ in Fig. 2). However, the mean-square longitudinal momentum $p_{\|}^{2}$ is still decreasing because electrons continue to lose energy to ions. As the amplitude of the magnetic field is too low to keep the plasma isotropic, $p_{\|}^{2}$ becomes lower than the mean-square transverse momentum $p_{\perp}^{2}$. In other words the plasma becomes again anisotropic, so that a new instability can develop (for $t \gtrsim 9$ fs in Fig. 2) [16]. Note that during this second growth the former hot axis $\boldsymbol{e}_{\boldsymbol{x}}$ is a cold axis.

In this paper, these two stages are analyzed in detail. First, the relevant dispersion relations are derived and discussed in Sec. II. Then the expansion is analyzed using twodimensional particle-in-cell (PIC) simulations in Sec. III.

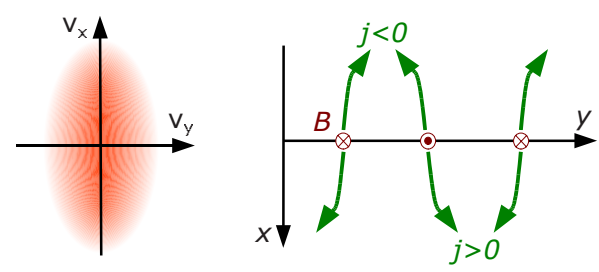

FIG. 1. (Color online) Principle of the Weibel instability. A perturbation magnetic field $\boldsymbol{B}$ drives a current $\boldsymbol{j}$, which enhances the original field. The arrows schematize the trajectories of the electrons which form the current sheets. 


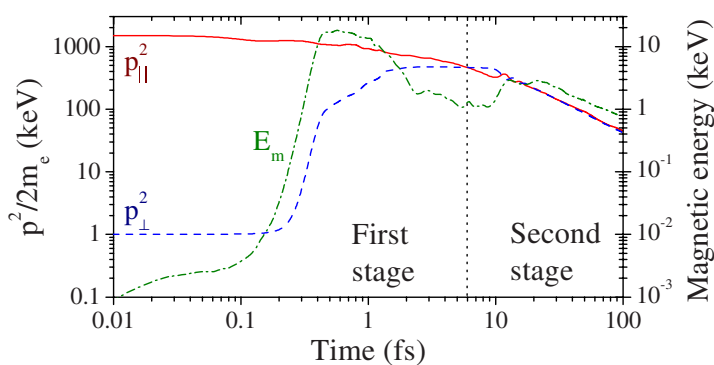

FIG. 2. (Color online) Energy transfers during the expansion of a plasma slab into a vacuum, in $\log -\log$ scale. The initial density and thickness of the slab are, respectively, $n_{e}=5 \times 10^{23} \mathrm{~cm}^{-3}$ and $L=150 \mathrm{~nm}$. At $t=0$ the plasma is nonmagnetized $\left[E_{m}(0)=0\right]$, and the mean-square longitudinal and transverse momenta are, respectively, $p_{\|}^{2} / 2 m_{e}=1.5 \mathrm{MeV}$ and $p_{\perp}^{2} / 2 m_{e}=1 \mathrm{keV}$. These results originate from a two-dimensional particle-in-cell simulation with periodic conditions in the transverse direction, and a transverse width of $180 \mathrm{~nm}$.

\section{DISPERSION RELATIONS}

In this section we establish dispersion relations for the Weibel instability in different conditions. We first assume in Sec. II A that all electrons have been accelerated to multi$\mathrm{keV}$ energies, so that the hot directions can be described by a single temperature, and consider successively the cases $p_{\|}^{2}$ $>p_{\perp}^{2}$ and $p_{\|}^{2}<p_{\perp}^{2}$. Then we discuss in Sec. II B the more realistic situation where only a part of the plasma electrons has been heated to high energies.

\section{A. Single population plasma \\ 1. Cold transverse temperature}

We assume here that the electron velocities are nonrelativistic and that the distribution function is of the form

$$
f^{(0)}(\boldsymbol{v}) \propto \exp \left[-\left|\boldsymbol{v}_{\perp}\right|^{2} / v_{\perp 0}^{2}-v_{\|}^{2} / v_{\| 0}^{2}\right] \quad \text { with } v_{\perp 0}<v_{\| 0}=v_{h 0} .
$$

We look for transverse magnetic modes with wave vector $\boldsymbol{k}$ parallel to $\boldsymbol{e}_{\boldsymbol{y}}$ and nonzero field components $\left(E_{x}, E_{y}, B_{z}\right)$. We express $f^{(0)}$ in the Cartesian basis $\left(\boldsymbol{e}_{\boldsymbol{x}}, \boldsymbol{e}_{\boldsymbol{y}}, \boldsymbol{e}_{z}\right)$, where $\boldsymbol{e}_{z}$ is chosen to be a cold direction, and where the hot direction lies in the $\left(\boldsymbol{e}_{\boldsymbol{x}}, \boldsymbol{e}_{\boldsymbol{y}}\right)$ plane such that the second cold direction makes an angle $\theta$ with respect to $\boldsymbol{k}$ [Fig. 3(a)]:

$$
f^{(0)}(\boldsymbol{v}) \propto \exp \left[-\left(v_{x}+\mu v_{y}\right)^{2} / v_{0}^{2}-v_{y}^{2} / v_{e}^{2}-v_{z}^{2} / v_{\perp 0}^{2}\right],
$$

with
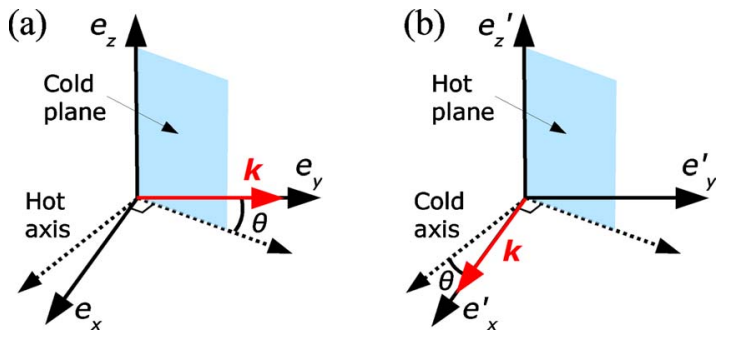

FIG. 3. (Color online) Standard basis used (a) when $p_{\|}^{2}>p_{\perp}^{2}$ (case of Sec. II A 1) and (b) when $p_{\|}^{2}<p_{\perp}^{2}$ (case of Sec. II A 2).

$$
\begin{gathered}
v_{e}^{2}=v_{h 0}^{2}\left(A \sin ^{2} \theta+1\right) /(A+1), \\
v_{0}^{2}=v_{h 0}^{2} /\left(A \sin ^{2} \theta+1\right), \\
\mu=A \cos \theta \sin \theta /\left(A \sin ^{2} \theta+1\right), \\
A=\left(v_{\| 0} / v_{\perp 0}\right)^{2}-1 .
\end{gathered}
$$

In this basis, the current $\boldsymbol{j}$ lies in the $\left(\boldsymbol{e}_{\boldsymbol{x}}, \boldsymbol{e}_{\boldsymbol{y}}\right)$ plane (it always builds in a hot direction) and $\boldsymbol{B}$ is along the $\boldsymbol{e}_{z}$ axis (it is parallel to $\boldsymbol{k} \times \boldsymbol{j}$ ).

To derive a dispersion relation for the unstable modes, we linearize the Maxwell and Vlasov equations for perturbations $\left(f^{(1)}, \boldsymbol{E}, \boldsymbol{B}\right) \sim \exp [i(k y-\omega t)]$ as follows:

$$
\begin{gathered}
B_{z}=-(k / \omega) E_{x}, \\
i c^{2} k B_{z}=j_{x} / \epsilon_{0}-i \omega E_{x}, \\
0=j_{y} / \epsilon_{0}-i \omega E_{y}, \\
-i \omega\left(1-\frac{v_{y} k}{\omega}\right) f^{(1)}=\frac{e}{m_{e}}\left[\left(E_{x}+v_{y} B_{z}\right) \frac{\partial f^{(0)}}{\partial v_{x}}\right. \\
\left.+\left(E_{y}-v_{x} B_{z}\right) \frac{\partial f^{(0)}}{\partial v_{y}}\right],
\end{gathered}
$$

where $\epsilon_{0}$ is the vacuum permittivity, $e$ is the electron charge, and $m_{e}$ is the electron mass. Combining Eqs. (2) and (3), and averaging $-e v_{x}$ over $f^{(1)}$, gives

$$
\begin{aligned}
\frac{i \omega j_{x}}{\epsilon_{0}}= & \left(c^{2} k^{2}-\omega^{2}\right) E_{x}=\omega_{p}^{2} \int d^{3} \boldsymbol{v} v_{x}\left[E_{x} \frac{\partial f^{(0)}}{\partial v_{x}}\right. \\
& \left.+\frac{\omega E_{y}+v_{x} k E_{x}}{\omega-v_{y} k} \frac{\partial f^{(0)}}{\partial v_{y}}\right],
\end{aligned}
$$

where $\omega_{p}=n_{e} e^{2} / m_{e} \epsilon_{0}$ is the electron plasma frequency. Similarly, combining Eqs. (2) and (4) and averaging $-e v_{y}$ over $f^{(1)}$ leads to

$$
\frac{i \omega j_{y}}{\epsilon_{0}}=-\omega^{2} E_{y}=\omega_{p}^{2} \int d^{3} \boldsymbol{v} \frac{v_{y}}{\omega-v_{y} k}\left[\omega E_{y}+v_{x} k E_{x}\right] \frac{\partial f^{(0)}}{\partial v_{y}} .
$$

Then, using integration by parts, Eqs. (6) and (7) become

$$
\begin{gathered}
-\left\langle\frac{v_{x} k \omega}{\left(\omega-v_{y} k\right)^{2}}\right\rangle E_{y}=\left[\frac{c^{2} k^{2}-\omega^{2}}{\omega_{p}^{2}}+1+\left\langle\frac{v_{x}^{2} k^{2}}{\left(\omega-v_{y} k\right)^{2}}\right\rangle\right] E_{x}, \\
{\left[\frac{\omega^{2}}{\omega_{p}^{2}}-\left\langle\frac{\omega^{2}}{\left(\omega-v_{y} k\right)^{2}}\right\rangle\right] E_{y}=\left\langle\frac{v_{x} k \omega}{\left(\omega-v_{y} k\right)^{2}}\right\rangle E_{x},}
\end{gathered}
$$



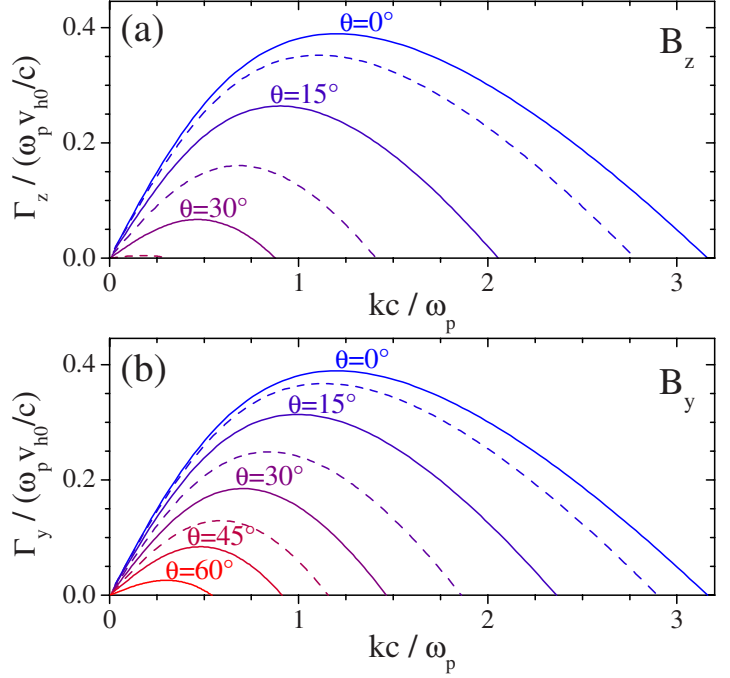

FIG. 4. (Color online) Growth rates of the Weibel instability as a function of the wave number, for different angles $\theta$ between $\boldsymbol{k}$ and the cold plane (or axis), $v_{h 0}=0.2 c$, and $A=10$. The angle $\theta$ is incremented by $7.5^{\circ}$ steps. In (a) the current lies in the anisotropic plane $\left(\boldsymbol{e}_{\boldsymbol{x}}, \boldsymbol{e}_{\boldsymbol{y}}\right)$, while in (b) it is along the $\boldsymbol{e}_{z}$ axis.

where the angular brackets denote the averaging over $f^{(0)}$. The dispersion relation is finally obtained by combining Eqs. (8) and (9),

$$
\begin{aligned}
c^{2} k^{2}= & \omega^{2}-\omega_{p}^{2}\left[1+\left\langle\frac{v_{x}^{2} k^{2}}{\left(\omega-v_{y} k\right)^{2}}\right\rangle\right. \\
& \left.-\left\langle\frac{v_{x} k \omega}{\left(\omega-v_{y} k\right)^{2}}\right\rangle^{2} /\left(\left\langle\frac{\omega^{2}}{\left(\omega-v_{y} k\right)^{2}}\right\rangle-\frac{\omega^{2}}{\omega_{p}^{2}}\right)\right] .
\end{aligned}
$$

Note that this equation can also be derived using the general expression of the dielectric tensor established in Ref. [23].

Purely growing solutions of Eq. (10), $\Gamma_{z}(k)=-i \omega(k)$, are plotted in Fig. 4(a) for different values of $\theta, A=10$, and $v_{h 0}$ $=0.2 c$. This figure shows that the most unstable modes are obtained for $\theta=0$, that is, when the wave vector lies in the cold plane. The maximum growth rate is divided by 2 when $\theta$ is increased from $0^{\circ}$ to $20^{\circ}$, and is reduced by almost two orders of magnitude when $\theta=37.5^{\circ}$.

The largest unstable (or critical) wave number $k_{c}$ is obtained by taking the limit $\omega \rightarrow 0$ in Eq. (10). This leads to

$$
\begin{aligned}
k_{c}^{2} c^{2} / \omega_{p}^{2} & =v_{0}^{2} / v_{e}^{2}-1+\mu^{2}[\pi / 2-1] \\
& =A \frac{\cos ^{2} \theta-\sin ^{2} \theta\left[1+A\left(1-\frac{\pi}{2} \cos ^{2} \theta\right)\right]}{\left(A \sin ^{2} \theta+1\right)^{2}} .
\end{aligned}
$$

For $A \rightarrow 0$, this equation reduces to $k_{c}^{2} c^{2} / \omega_{p}^{2}$ $\approx A\left(1-2 \sin ^{2} \theta\right)$, which shows that for small $A$, purely growing modes are obtained only for $\theta<45^{\circ}$. Similarly, taking the limit $A \rightarrow \infty$ for $\theta \neq 0$ in Eq. (11) leads to $k_{c}^{2} c^{2} / \omega_{p}^{2}$ $\approx\left[(\pi / 2) \cos ^{2} \theta-1\right] / \sin ^{2} \theta$. In this case, $k_{c}$ does not depend on $A$ and is much smaller than for $\theta=0$ where $k_{c}^{2} c^{2} / \omega_{p}^{2}=A$.
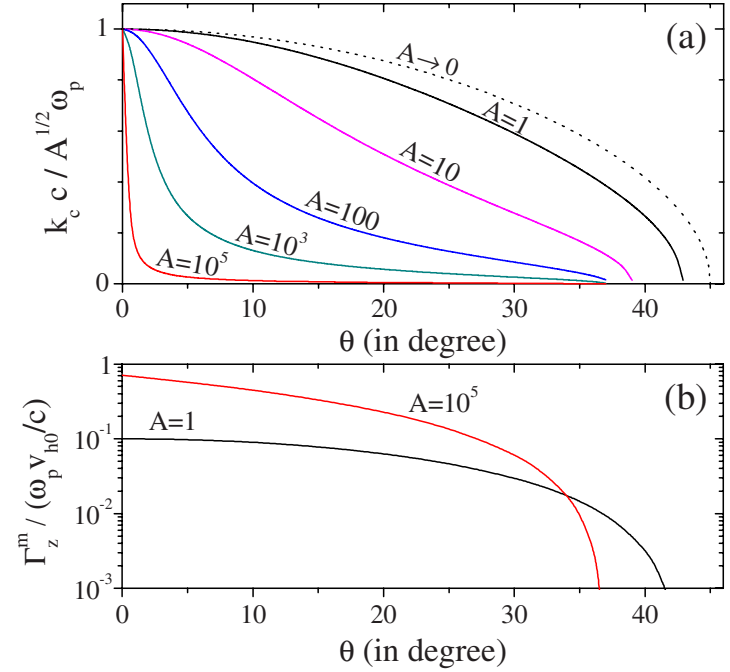

FIG. 5. (Color online) Influence of $\theta$ on the largest unstable wave vector and on the maximum growth rate for $B_{z}$. (a) Largest unstable wave number $k_{c}$ normalized to $\sqrt{A} \omega_{p} / c$, as a function of $\theta$, for different anisotropy parameters $A$. The dotted line corresponds to the limit $A \rightarrow 0$. (b) Maximum growth rate as a function of $\theta$ for $A=1$ and $A=10^{5}$, in a logarithmic scale.

All these findings are confirmed in Fig. 5(a) which displays $k_{c}(\theta)$ for five different values of $A$.

Figure 5(b) shows that the maximum growth rate $\Gamma_{z}^{m}$ also decreases more steeply with $\theta$ for large $A$. As a result of the behavior of both $k_{c}$ and $\Gamma_{z}^{m}$, unstable modes for large $A$ grow mainly with wave vector in the cold plane. In contrast, for small $A$ and $\theta \leqq 10^{\circ}, k_{c}$ and $\Gamma_{z}^{m}$ depend weakly on $\theta$, so modes can grow efficiently with $0<\theta \leq 10^{\circ}$.

\section{Hot transverse temperature}

We turn now to the case where the electron distribution function is of the form

$$
f^{(0)}(\boldsymbol{v}) \propto \exp \left[-\left|\boldsymbol{v}_{\perp}\right|^{2} / v_{\perp 0}^{2}-v_{\|}^{2} / v_{\| 0}^{2}\right] \quad \text { with } v_{\perp 0}=v_{h 0}>v_{\| 0} .
$$

This corresponds to the second stage of the plasma expansion in Fig. 2.

We first express $f^{(0)}$ in the standard basis $\left(\boldsymbol{e}_{x}^{\prime}, \boldsymbol{e}_{y}^{\prime}, \boldsymbol{e}_{z}^{\prime}\right)$ in which $\boldsymbol{e}_{z}^{\prime}$ is a hot axis and $\boldsymbol{k}=\boldsymbol{k} \boldsymbol{e}_{\boldsymbol{x}}^{\prime}$ :

$$
f^{(0)}(\boldsymbol{v}) \propto \exp \left[-\left(v_{y}+\mu v_{x}\right)^{2} / v_{0}^{2}-v_{x}^{2} / v_{e}^{2}-v_{z}^{2} / v_{\perp 0}^{2}\right],
$$

where $v_{e}, v_{0}$, and $\mu$ have the same definitions as in Eq. (1), except that $A=\left(v_{\perp 0} / v_{\| 0}\right)^{2}-1$, and $\theta$ is the angle between the wave vector $\boldsymbol{k}$ and the cold axis or, equivalently, the angle between $\boldsymbol{e}_{y}^{\prime}$ and the hot plane [see Fig. 3(b)]. In this situation, $\boldsymbol{B}$ must lie in the $\left(\boldsymbol{e}_{y}^{\prime}, \boldsymbol{e}_{z}^{\prime}\right)$ plane. One possible combination of nonzero fields is $\left(E_{x}, E_{y}, B_{z}\right)$. This case is identical to the one studied in Sec. II A 1, just exchanging the roles of $x$ and $y$ in the calculations. A second combination is $\left(E_{z}, B_{y}\right)$. We note that in this case, where the cold direction and $\boldsymbol{k}$ are both 
perpendicular to $\boldsymbol{e}_{z}^{\prime}$, an electric field in the $\boldsymbol{e}_{z}^{\prime}$ direction produces no current density in the $\left(\boldsymbol{e}_{x}^{\prime}, \boldsymbol{e}_{y}^{\prime}\right)$ plane, due to the reflection symmetry of the distribution function in $\boldsymbol{e}_{z}^{\prime}$. Consequently, $E_{x}=E_{y}=0$.

As in Sec. II A 1 we begin by writing the linearized Maxwell and Vlasov equations,

$$
\begin{aligned}
& B_{y}=-(k / \omega) E_{z}, \\
& i c^{2} k B_{y}=j_{z} / \epsilon_{0}-i \omega E_{z}, \\
&-i \omega\left(1-\frac{v_{x} k}{\omega}\right) f^{(1)}=\frac{e}{m_{e}}\left[\left(E_{x}-v_{z} B_{y}\right) \frac{\partial f^{(0)}}{\partial v_{x}}\right. \\
&\left.+\left(E_{z}+v_{x} B_{y}\right) \frac{\partial f^{(0)}}{\partial v_{z}}\right] .
\end{aligned}
$$

Then we combine Eqs. (13) and (14), and average $-e v_{z}$ over $f^{(1)}$, to get

$$
\begin{aligned}
\frac{i \omega j_{z}}{\epsilon_{0}} & =\left(c^{2} k^{2}-\omega^{2}\right) E_{z} \\
& =\omega_{p}^{2} \int d^{3} \boldsymbol{v}\left[\frac{k v_{z}^{2}}{\omega-v_{x} k} E_{z} \frac{\partial f^{(0)}}{\partial v_{x}}+v_{z} E_{z} \frac{\partial f^{(0)}}{\partial v_{z}}\right] .
\end{aligned}
$$

We finally apply integration by parts to obtain the following dispersion relation:

$$
\begin{aligned}
c^{2} k^{2} & =\omega^{2}-\omega_{p}^{2}\left[1+\frac{k^{2}}{2}\left\langle\frac{v_{z}}{\left(\omega-v_{x} k\right)^{2}}\right\rangle\right] \\
& =\omega^{2}-\omega_{p}^{2}\left[1-\frac{A+1}{A \sin ^{2} \theta+1}[1+\xi Z(\xi)]\right],
\end{aligned}
$$

where $\xi=\omega / k v_{e}$ and $Z(\xi)=\pi^{-1 / 2} \int d t \exp \left[t^{-2}\right] /(t-\xi)$ is the plasma dispersion function [24].

The growth rate $\Gamma_{y}(k)$ obtained from Eq. (17) is plotted in Fig. 4(b). For $\theta=0$, the growth rates are the same for $B_{y}$ and $B_{z}$ [Fig. 4(a)]. In contrast, for $\theta>0, \Gamma_{y}(k)$ is larger than $\Gamma_{z}(k)$. The reason for that is that when $\boldsymbol{B}$ grows along the $\boldsymbol{e}_{\boldsymbol{y}}^{\prime}$ axis the currents are purely along a hot direction, which is not the case when it grows along the $\boldsymbol{e}_{z}^{\prime}$ axis (except for $\theta$ $=0$ ); currents are thus larger in the first case.

The critical wave number,

$$
k_{c}^{2} c^{2} / \omega_{p}^{2}=\frac{A \cos ^{2} \theta}{A \sin ^{2} \theta+1},
$$

is also larger for $B_{y}$ than for $B_{z}$ [compare Eqs. (11) and (19) and the two panels of Fig. 4]. Another difference is that purely growing modes are obtained for all $\theta<90^{\circ}$ when $\boldsymbol{B}$ is along the $\boldsymbol{e}_{y}^{\prime}$ axis. In contrast the influence of $A$ on $k_{c}$ is similar in both cases. Indeed, Eq. (19) shows that $k_{c}^{2} c^{2} \omega_{p}^{2}$ $\approx A \cos ^{2} \theta$ when $A \rightarrow 0$, and $k_{c}^{2} c^{2} \omega_{p}^{2} \approx \cot ^{2} \theta$ when $A \rightarrow \infty$ and $\theta \neq 0$.

\section{Discussion for $\theta=0$}

Sections II A 1 and II A 2 show that, in all cases, the most unstable mode grows with a wave vector in a cold direction.
It is therefore interesting to study in detail the dispersion relation for $\theta=0$. Note that even though the most unstable modes are always obtained for $\theta=0$, for small anisotropy, $k_{c}$ and $\Gamma^{m}$ decrease slowly when $\theta$ is increased (see Fig. 5). Modes with $\theta>0$ can therefore develop during the second stage of the expansion during which the anisotropy is quite small.

In this section we assume that $\theta=0$ and we derive a simple expression for $\Gamma^{m}$ when the anisotropy is large. Then we discuss the case of relativistic velocities. The starting point for this study is the dispersion relation obtained by taking $\theta=0$ in Eq. (10) or Eq. (17):

$$
c^{2} k^{2}=\omega^{2}+\omega_{p}^{2}[A+(A+1) \xi Z(\xi)],
$$

with $\xi=\sqrt{A+1} \omega / k v_{h 0}$. As mentioned before, the largest unstable wave vector in this case is $k_{c}=\sqrt{A} \omega_{p} / c$.

(a) Limit for large anisotropy. When $A$ is large, $Z(\xi)$ can be substituted by its asymptotic expansion for $|\xi| \gg 1, Z(\xi)$ $\approx-\xi^{-1}\left(1+1 / 2 \xi^{2}+3 / 4 \xi^{4}\right)[25]$, so that Eq. (20) becomes

$$
c^{2} k^{2} \approx-\Gamma^{2}+\omega_{p}^{2}\left[-1+\frac{k^{2} v_{h 0}^{2}}{2} \Gamma^{-2}-\frac{3 k^{4} v_{h 0}^{4}}{4(A+1)} \Gamma^{-4}\right] \text {. }
$$

Differentiating Eq. (21) with respect to $k$, we find that for $A \gg 1$ the maximum growth rate is

$$
\begin{aligned}
\Gamma^{m} & \approx\left[1-\sqrt{\frac{6\left(2+v_{h 0} / c\right)}{A}}\right]^{1 / 2} \frac{v_{h 0}}{\sqrt{2} c} \omega_{p} \\
& \approx \frac{v_{h 0}}{\sqrt{2} c} \omega_{p} \quad \text { when } A \rightarrow \infty .
\end{aligned}
$$

Then, combining Eqs. (21) and (22), we find the wave number of the most unstable mode,

$$
k^{m} \approx \frac{\omega_{p}}{c}\left[\frac{2+v_{h 0} / c}{6} A\right]^{1 / 4} \approx \frac{\omega_{p}}{c}\left[\frac{A}{3}\right]^{1 / 4} \text { for } v_{h 0} \ll c .
$$

Equation (22) shows that, for large $A, \Gamma^{m}$ is almost a linear function of the hot velocity. It indicates also that $\Gamma^{m}$ saturates when $A \rightarrow \infty$. Further Eq. (23) states that the wave number of the most unstable mode $k^{m}$ increases much more slowly with $A$ than the critical wave number $k_{c}=\sqrt{A} \omega_{p} / c$. This means that the instability develops mainly with relatively small wave numbers (for $A=10000, k^{m} \approx 7.6 \omega_{p} / c$, while $\left.k_{c}=100 \omega_{p} / c\right)$. Note that this is not the case for $A \ll 1$, where $k^{m}=k_{c} / \sqrt{3}$. In summary for large anisotropy, the growth rate saturates and the most unstable wave number increases slowly with $A$ (more slowly that $k_{c}$ ).

(b) Relativistic velocities. For now this study is quite academic. In an experiment the velocity is generally relativistic when the anisotropy is very large. It is thus necessary to analyze how the previous results are modified when $v_{h 0} \rightarrow c$. As it is much more complicated to derive a dispersion relation in this case [26-28], we limit the present discussion to an analysis of one spatial and three velocity dimensions $(1 \mathrm{D} 3 \mathrm{~V})$ relativistic PIC simulations. In these simulations, the initial distribution function is $f^{(0)} \propto \exp \left[-\left(\gamma_{x}-1\right) /\left(\gamma_{\| 0}-1\right)\right.$ 


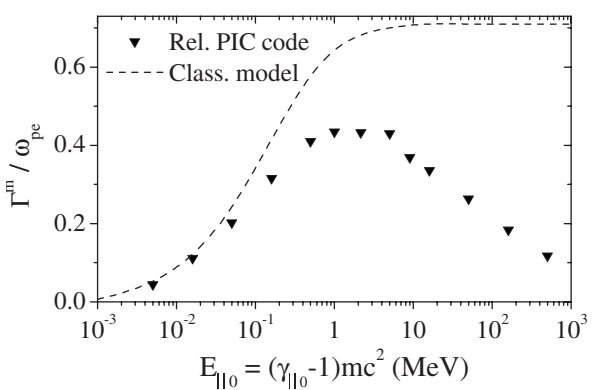

FIG. 6. Growth rates for relativistic thermal velocities. The triangles are the results of $1 \mathrm{D} 3 \mathrm{~V}$ relativistic PIC simulations performed for a mean-square transverse momentum $p_{\perp 0}^{2} / 2 m_{e}$ $=500 \mathrm{eV}$. The dashed line is the maximum growth rate provided by Eq. (20).

$\left.-\left(\gamma_{y}+\gamma_{z}-2\right) /\left(\gamma_{\perp 0}-1\right)\right]$ with $\gamma_{x}=\left(1-v_{x}^{2} / c^{2}\right)^{-1 / 2}$ and $\gamma_{0 \|}=(1$ $\left.-v_{\| 0}^{2} / c^{2}\right)^{-1 / 2}$ (and so on for $\gamma_{y}, \gamma_{z}$, and $\gamma_{0 \perp}$ ). The wave vector is necessarily along $\boldsymbol{e}_{\boldsymbol{y}}$, which is the only spatial dimension in these $1 \mathrm{D}$ simulations, so $\theta$ is forced to zero. Ions are fixed and the width of the plasma along the $\boldsymbol{e}_{\boldsymbol{y}}$ axis is $42 c / \omega_{p}$ (see Sec. III A 1 for supplementary information on the simulations).

Figure 6 displays the results of simulations performed for different values of $E_{\| 0}=\left(\gamma_{\| 0}-1\right) m c^{2}$, and a mean-square transverse momentum $p_{\perp 0}^{2} / 2 m_{e}=500 \mathrm{eV}$. For $E_{\| 0} \lesssim 50 \mathrm{keV}$, the maximum growth rate $\Gamma^{m}$ is almost equal to the one given by Eq. (20). For larger longitudinal energies, the simulation results start to differ significantly from the classical model. The growth rate saturates for $E_{\| 0} \approx 1 \mathrm{MeV}\left(v_{\| 0}\right.$ $\approx 0.94 c$ ) as in the classical limit, but the value reached by $\Gamma^{m}$ is lower in the relativistic regime $\left(0.43 \omega_{p}\right.$, instead of $\left.0.71 \omega_{p}\right)$. Note that this value is not modified when $p_{\perp 0}^{2}$ is reduced. The most important difference is however observed for $E_{\| 0}>5 \mathrm{MeV}$, where $\Gamma^{m}$ exhibits a slow decay.

To explain this behavior, we derive a dispersion relation for the simplest relativistic distribution function $f \propto \delta\left(\gamma_{x}\right.$ $\left.-\gamma_{\| 0}\right) \delta\left(v_{y}\right) \delta\left(v_{z}\right)$, where $\delta(x)$ is the Dirac delta function. Using the general dispersion relation established in Ref. [23], we obtain after integration,

$$
c^{2} k^{2}+\Gamma^{2}+\omega_{p}^{2} \gamma_{\| 0}^{-3}\left[1-c^{2} k^{2}\left(\gamma_{\| 0}^{2}-1\right) \Gamma^{-2}\right]=0 .
$$

Then, differentiating Eq. (24) we find that the maximum growth rate, for $\gamma_{\| 0} \gg 1$, is $\Gamma_{r}^{m}=\omega_{p} / \sqrt{\gamma_{\| 0}}$. In agreement with Fig. 6 this growth rate decreases when the longitudinal energy is raised. Physically, this originates from the relativistic increase of the mass, which makes electrons harder to move in the $\boldsymbol{e}_{\boldsymbol{y}}$ direction when $\gamma_{\| 0}$ increases. Note that this effect is also observed analytically when more realistic distribution functions are used [27].

To sum up this section, the growth rate of the most unstable mode $\Gamma^{m}$ increases with $A$ before saturating for very large anisotropy. This property which has been demonstrated for nonrelativistic thermal velocities is also verified when $v_{h 0}$ is relativistic. Additional PIC simulations actually show that for $E_{\| 0}=50 \mathrm{MeV}, \Gamma^{m}$ varies by less than $1 \%$ when $10^{3} \leq A$ $\leq 10^{5}$. In the classical regime $\left(E_{\| 0} \leqq 50 \mathrm{keV}\right)$, the maximum growth rate rises almost linearly with the thermal velocity in
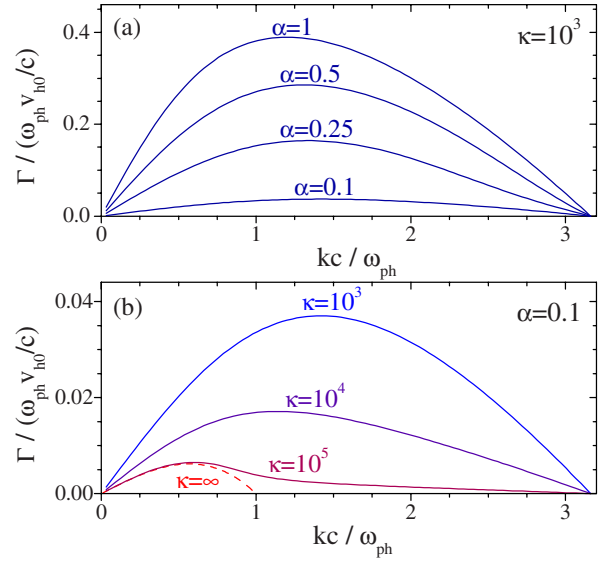

FIG. 7. (Color online) Dispersion relations in the presence of cold electrons for $A=10$ and $v_{h 0}=0.2 c$. (a) Growth rate $\Gamma /\left(\omega_{p h} v_{h 0} / c\right)$ as a function of $k c / \omega_{p h}$ for $\kappa=\left(v_{h 0} / v_{c 0}\right)^{2}=10^{3}$ and different fractions of hot electrons $\alpha$. (b) Influence of $\kappa$ on the dispersion curve for $\alpha=0.1$. The dashed line corresponds to the limit $\kappa \rightarrow \infty$.

the hot direction. In contrast, in the relativistic regime, $\Gamma^{m}$ is observed to decrease when the longitudinal energy is increased above approximately $5 \mathrm{MeV}$. As a result, the optimal growth rate is obtained for $E_{\| 0} \approx 1-5 \mathrm{MeV}$. This growth rate can however be much smaller than predicted in Fig. 6 if the plasma contains a large population of cold electrons.

\section{B. Two population plasma}

In this section, we analyze the influence on the Weibel instability of a cold isotropic population in the classical limit. This case is of particular interest in the context of laserplasma interaction, where the laser heats only a part of the plasma electrons to very high energies. To investigate the impact of a cold population, we consider distribution functions of the form

$$
f_{2 p}^{(0)}(\boldsymbol{v})=\alpha f_{h}^{(0)}(\boldsymbol{v})+(1-\alpha) f_{c}^{(0)}(\boldsymbol{v}),
$$

with $\alpha=n_{h 0} /\left(n_{h 0}+n_{c 0}\right)$ as the fraction of hot electrons $\left(n_{h 0}\right.$ and $n_{c 0}$ are, respectively, the hot and cold electron densities), $f_{h}^{(0)}=f^{(0)}$, and $f_{c}^{(0)}(\boldsymbol{v}) \propto \exp \left[-|\boldsymbol{v}|^{2} / v_{c 0}^{2}\right]$.

For simplicity we assume that $\theta=0$, so that we can easily derive from Eq. (17) the dispersion relation for the two population plasma,

$$
c^{2} k^{2}=\omega^{2}+\omega_{p h}^{2}[A+(A+1) \xi Z(\xi)]+\frac{1-\alpha}{\alpha} \omega_{p h}^{2} \xi_{c} Z\left(\xi_{c}\right),
$$

with $\omega_{p h}^{2}=\alpha \omega_{p}^{2}$ and $\xi_{c}=\omega / k v_{c 0}$. Taking the limit $\omega=0$, we find that the critical wave number is $k_{c}=\sqrt{A} \omega_{p h} / c$. This suggests that the presence of a cold population does not perturb much the growth of the instability. But Fig. 7(a), which displays the growth rate of purely growing modes for different hot electron fractions, demonstrates that this conclusion is not correct since $\Gamma / \omega_{p h}$ is observed to decrease with $\alpha$. Further, Fig. 7(b) shows that $\Gamma / \omega_{p h}$ is also reduced when the 

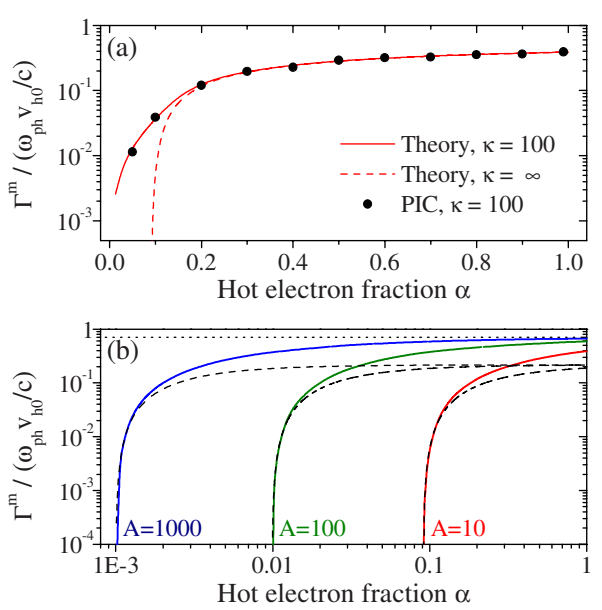

FIG. 8. (Color online) Influence of the hot electron fraction $\alpha$ on the maximum growth rate $\Gamma^{m}$. In (a), the model for $A=10$ and $\kappa$ $=100$ is compared to $1 \mathrm{D} 3 \mathrm{~V}$ PIC simulations with fixed ions. The theoretical growth rate obtained for $\kappa \rightarrow \infty$ is also plotted in dashed line. In (b) $\Gamma^{m}$ is plotted as a function of $\alpha$ for three different values of $A$. The dotted line corresponds to the limit $A \rightarrow \infty$ and the dashed lines correspond to the limits obtained when $\alpha A \approx 1$.

ratio $\kappa=\left(v_{h 0} / v_{c 0}\right)^{2}$ is increased, i.e., when the cold electrons become colder or, equivalently, when the hot electrons become hotter (while $A$ is kept constant). Finally, we remark that the wave number of the most unstable mode shifts to lower value when $\kappa$ rises.

To understand these different effects, we take the limit $v_{c 0} \rightarrow 0$ in Eq. (26) to obtain another dispersion relation,

$$
c^{2} k^{2}=\omega^{2}+\omega_{p h}^{2}[1-1 / \alpha+A+(A+1) \xi Z(\xi)] .
$$

The purely growing solution of Eq. (27) is plotted in Fig. 7 (dashed line). We see that the growth rate $\Gamma^{m}$ is 200 lower in this case than in the single population case. Besides, the critical wave vector,

$$
k_{c}=\frac{\omega_{p h}}{c} \sqrt{A+1-\frac{1}{\alpha}},
$$

is smaller than in the finite- $\kappa$ case. This means that for very low cold thermal velocity, the growth rate for $\sqrt{A+1-1 / \alpha}$ $<k^{m} c / \omega_{p h}<\sqrt{A}$ tends to zero. Thus, the instability is almost suppressed when $\alpha<1 /(A+1)$. In other words, cold electrons can stabilize the plasma if their fraction is sufficiently high.

This is illustrated in Fig. 8(a) which shows that the maximum growth rate $\Gamma^{m}$ decreases strongly with $\alpha$ when $\alpha$ $\lesssim 1 /(A+1)$, even when $\kappa$ is relatively small. It also reveals a very good agreement between Eq. (26) and 1D PIC simulations with fixed ions, thus validating the theoretical predictions. Further, Fig. 8(a) shows that for $\alpha \gg 1 /(A+1)$ the solution obtained for $\kappa \rightarrow \infty$ is very close to the one given by Eq. (26). As Fig. 7 proves, in addition, that Eq. (27) provides a good estimate of $\Gamma^{m}$ when $\kappa>10^{5}$, even for $\alpha \approx 1 / A$, we can use it - as a first approximation-to study the influence of the cold population.
To explain the "stabilization" by the cold population, we rewrite Eq. (27) to make appear the cold plasma frequency $\omega_{p c}^{2}=(1-\alpha) \omega_{p}^{2}$,

$$
c^{2} k^{2}=\omega^{2}-\omega_{p c}^{2}+\omega_{p h}^{2}[A+(A+1) \xi Z(\xi)] .
$$

We see that the plasma is stabilized if $\omega_{p c}^{2} \gg \omega_{p h}^{2}[A+(A$ $+1) \xi Z(\xi)]$, that is, if the cold population response to the perturbation is much stronger than the one of the hot population. To elucidate the influence of $v_{c 0}$, we take one more term in the Taylor expansion of $Z\left(\xi_{c}\right)$ in Eq. (26), replacing $\omega_{p c}^{2}$ with $\omega_{p c}^{2}\left(1-k^{2} v_{c 0}^{2} / 2 \Gamma^{2}\right)$ in Eq. (29). Thus we see that a finite velocity $v_{c 0}$ tends to reduce the influence of cold electrons. In fact the cold return current associated with the $\omega_{p c}^{2}$ term is the same whether $v_{c 0}=0$ or $v_{c 0} \neq 0$, but in the latter case electrons are also subjected to the magnetic Lorentz force which drives currents in the opposite direction, and hence reduces the influence of cold electrons. The impact of this second term increases with $v_{c 0}$.

We study now the influence of the anisotropy parameter. For $\alpha A \gg 1$, we find, in the same way that we obtained Eq. (22), that the maximum growth rate is

$$
\Gamma^{m} \approx\left[1-\sqrt{\frac{6\left(2+\alpha v_{h 0} / c\right)}{\alpha A}}\right]^{1 / 2} \frac{v_{h 0}}{\sqrt{2} c} \omega_{p} \approx \frac{v_{h 0}}{\sqrt{2} c} \omega_{p} .
$$

The maximum growth rate is thus almost unchanged by the presence of cold electrons if $\alpha A$ is sufficiently large. In contrast, when $\alpha A \approx 1, \xi Z(\xi) \approx-\sqrt{\pi(A+1)} \Gamma / k v_{h 0}$ and Eq. (27) leads to

$$
\Gamma^{m} \approx \frac{1}{\sqrt{\pi}}\left(\frac{2}{3}\right)^{3 / 2}\left[1-\frac{1}{\alpha(A+1)}\right]^{3 / 2} \frac{v_{h 0}}{\sqrt{2} c} \omega_{p h},
$$

which confirms that $\Gamma^{m}$ strongly decreases with $\alpha$ when $\alpha$ $\rightarrow(A+1)^{-1}$, whatever is the anisotropy. To illustrate these effects, we plotted in Fig. 8(b) $\Gamma^{m}$ as a function of $\alpha$ for three different anisotropy parameters (solid lines). We observe that Eqs. (30) and (31) provide a good approximation of $\Gamma^{m}$ for, respectively, large and small $\alpha A$. For large $A$, the influence of cold electrons is very weak if $\alpha \gg 1 / A$, while the instability can be suppressed, even for very high anisotropy, if the plasma is mainly made of cold electrons.

To conclude this section, we address the specific case of a cold population with a temperature equal to the cold axis temperature $\left[v_{c 0}^{2}=v_{h 0}^{2} /(A+1)\right]$. This case may be of importance in the context of laser heated plasma foils, where such a distribution is obtained when only a part of the plasma electrons gains energy from the laser and is accelerated in a preferential direction. In this situation, $\xi_{c}=\xi$ and Eq. (26) becomes

$$
\begin{aligned}
c^{2} k^{2}= & \omega^{2}-\omega_{p c}^{2}+\omega_{p h}^{2}\left[A^{\prime}+\left(A^{\prime}+1\right) \xi Z(\xi)\right] \\
& \text { with } A^{\prime}=A+(1-\alpha) / \alpha
\end{aligned}
$$

Comparing Eqs. (29) and (32) reveals that this dispersion relation is the same as the one obtained for an infinitely cold population substituting $A$ by $A^{\prime}$. Thus, the previous analysis, and in particular Eqs. (30) and (31), can be used in this case, 
without approximation, by just replacing $A$ with $A^{\prime}$. For instance, we get for small $\alpha A$

$$
\Gamma^{m} \approx \frac{1}{\sqrt{\pi}}\left(\frac{2}{3}\right)^{3 / 2}\left[1-\frac{1}{\alpha A+1}\right]^{3 / 2} \frac{v_{h 0}}{\sqrt{2} c} \omega_{p h} .
$$

Note that, in contrast with Eq. (31), $\Gamma^{m}>0$ for all $\alpha A>0$.

\section{SIMULATIONS OF THE PLASMA EXPANSION}

Up to now, we have studied the development of the Weibel instability in a plasma with fixed ions. To take into account the influence of the expansion, we perform in this section two spatial and three velocity dimensions (2D3V) PIC simulations with mobile ions, and use the results of Sec. II to analyze the numerical findings. First we present in Sec. III A simulations with an initial anisotropy and without laser pulse, and then we discuss in Sec. III B a simulation without initial anisotropy but with a laser pulse focused on the plasma slab.

\section{A. Two temperature expanding plasmas}

\section{Numerical conditions}

The PIC code used in this study is relativistic and noncollisional. It involves two spatial $\left(\boldsymbol{e}_{\boldsymbol{x}}\right.$ and $\left.\boldsymbol{e}_{\boldsymbol{y}}\right)$ and three velocity dimensions. The simulation box size is $1000 c / \omega_{p h}$ $\times 24 c / \omega_{p h}$. The plasma is a foil with an initial thickness along the $\boldsymbol{e}_{x}$ (longitudinal) axis of $L=20 c / \omega_{p h}$. The boundary conditions in the transverse direction are periodic for both fields and particles. In the longitudinal direction, open boundaries are used for the fields while electrons are reflected. The time step is $\Delta t=0.1 \omega_{p}^{-1}$ and the spatial grid sizes are $\Delta x=\Delta y=0.16 \mathrm{c} / \omega_{p}$. The ion to electron mass ratio is $m_{i} / m_{e}=1836$, and the ion charge is $Z=1$.

In this section we consider plasmas with an initial meansquare longitudinal momentum $p_{\| 0}^{2}$ larger than the transverse one $p_{\perp 0}^{2}$, and with or without a cold isotropic electron population. The transverse and the cold population temperatures are both of $1 \mathrm{keV}$, while the ion temperature is $30 \mathrm{eV}$. To limit both the computation time and the noise level, we take in our simulations $\alpha \geq 0.1$, which is sufficient to analyze the influence of cold electrons. There are initially $\approx 9 \times 10^{6}$ hot macroparticles when $\alpha=1$, and $\approx 3 \times 10^{6}$ when $\alpha=0.1$.

\section{Simulation results}

Figures 9 and 10 are the results of 2D3V PIC simulations performed for different initial hot longitudinal energies $E_{h 0}$ $=\left(\gamma_{h 0}-1\right) m_{e} c^{2}$ and different fractions of hot electrons $\alpha$. They display, respectively, the mean-square longitudinal and transverse momenta and the mean magnetic energy as functions of time (Fig. 9), and the magnetic field as a function of space for different times (Fig. 10).

(a) Nonrelativistic case. In Figs. 9(a) and 10(a), $\alpha=1$ and $E_{h 0}=10 \mathrm{keV}(A=9)$. The magnetic energy is observed to increase with a growth rate $2 \Gamma_{P I C} \approx 2 \times 0.055 \omega_{p h}$, before saturating at $t \approx 100 \omega_{p h}^{-1}$ [Fig. 9(a)]. According to the linear theory of Sec. II, the maximum growth rate in this nonrelativistic case should be $\Gamma^{m}=0.053 \omega_{p h}$. The theoretical and nu-

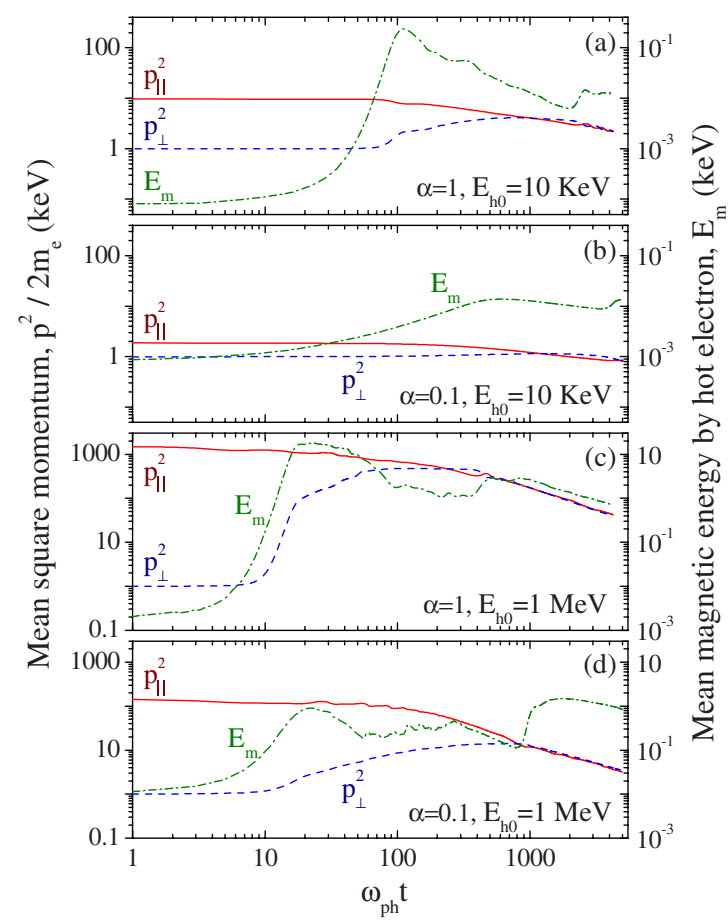

FIG. 9. (Color online) Mean magnetic energy by hot electron, and mean-square transverse and longitudinal momenta as functions of time, in log-log scale, for different hot fractions $\alpha$ and hot longitudinal energies $E_{h 0}=\left(\gamma_{h 0}-1\right) m_{e} c^{2}$.

merical values are very close, which indicates that the linear model describes correctly the development of the Weibel instability, even for a two-dimensional expanding plasma. Figure 10(a) shows the spatial variations of the magnetic field, when it reaches its maximum at $t \approx 100 \omega_{p h}^{-1}$. In agreement with Sec. II A 1, the wave vector lies in the transverse plane $(\theta=0)$. Further, the value of the most unstable wave number $k_{P I C}^{m} \approx 1.3 \omega_{p h} / c$ is also close to the value predicted by the linear model $\left(k^{m}=1.2\right)$, which confirms its accuracy. Note that at earlier times there are several other unstable modes which eventually coalesce $[29,30]$.

Figure 9(a) shows that, due to the magnetic field, electrons transfer a part of their longitudinal momentum to the transverse direction up to $t \approx 1000 \omega_{p h}^{-1}$, where $p_{\|}^{2}=p_{\perp}^{2}(A$ $=0)$. At this time, the mean-square transverse momentum stops rising, while the longitudinal one continues decreasing because electrons lose their energy to ions. As the magnetic field at $t \approx 1000 \omega_{p h}^{-1}$ is not intense enough to isotropize "instantaneously" the electron distribution, the anisotropy $A$ $\approx p_{\perp}^{2} / p_{\|}^{2}-1$ grows. The maximum anisotropy reached during this second stage is $A \approx 0.12$, which is sufficient to drive a new Weibel instability. Indeed, for $A \approx 0.12$ we have $k_{c}$ $\approx 0.35 \omega_{p h} / c$, while the smallest unstable wave number which can be sustained by the plasma is $k=2 \pi / L$ $\approx 0.31 \omega_{p h} / c<k_{c}$. Note that for simplicity the plasma expansion is neglected in this analysis. A more comprehensive discussion can be found in Ref. [16].

As a consequence of this second instability, the magnetic energy restarts to rise and it reaches a second maximum at $t \approx 2700 \omega_{p h}^{-1}$. The magnetic field at this time is plotted in Fig. 10(a) (right column). The wave vector of the most unstable 

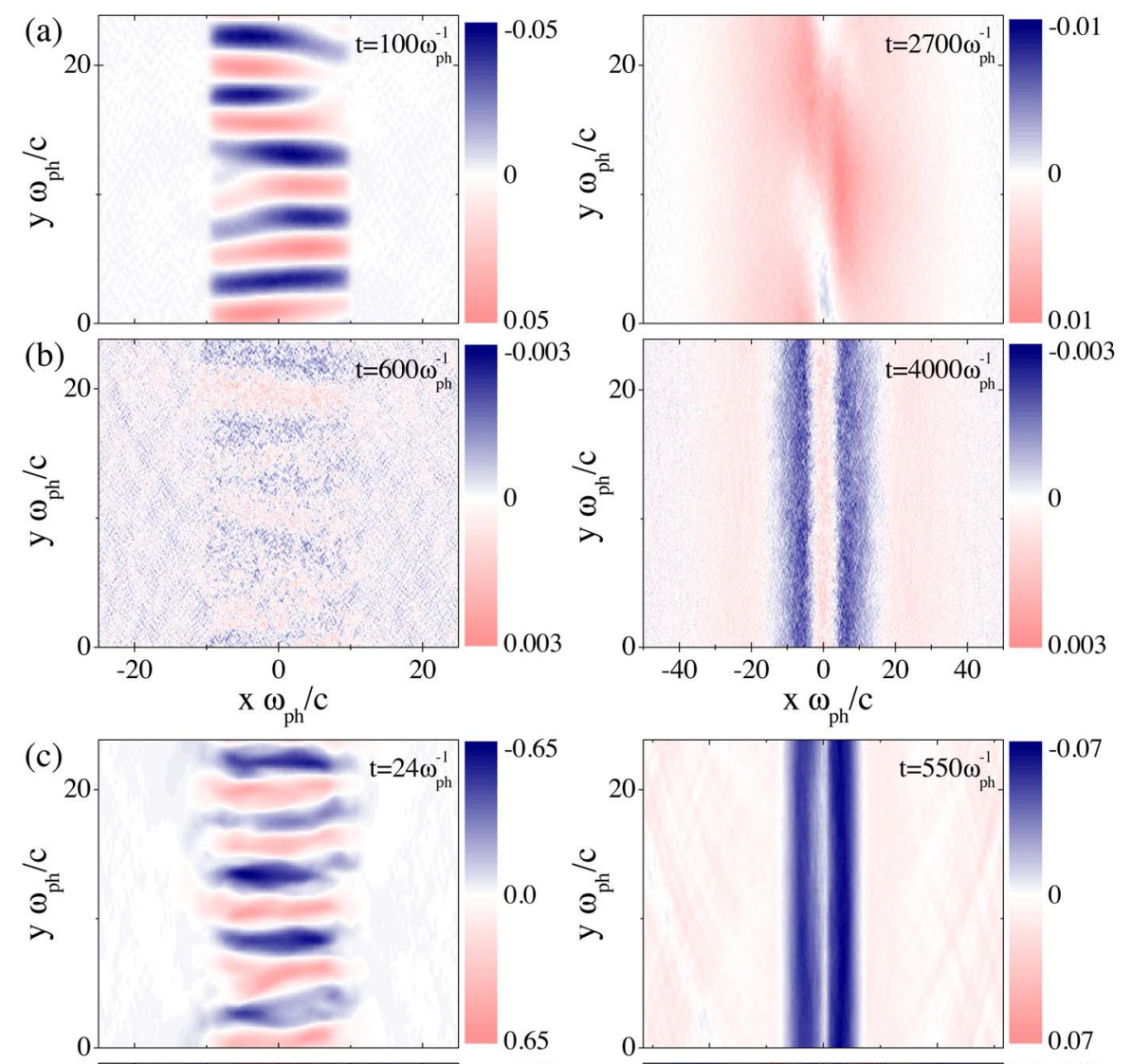

FIG. 10. (Color online) Magnetic field $B_{z}(x, y)$ at different times in unit of $m_{e} \omega_{p h} / e$. In (a) $E_{h 0}=10 \mathrm{keV}$ and $\alpha=1$. In (b) $E_{h 0}=10 \mathrm{keV}$ and $\alpha=0.1$. In (c) $E_{h 0}=1 \mathrm{MeV}$ and $\alpha=1$. In (d) $E_{h 0}=1 \mathrm{MeV}$ and $\alpha=0.1$. The fields in the left column have developed during the first stage of the expansion, while those in the right column have developed during the second stage.
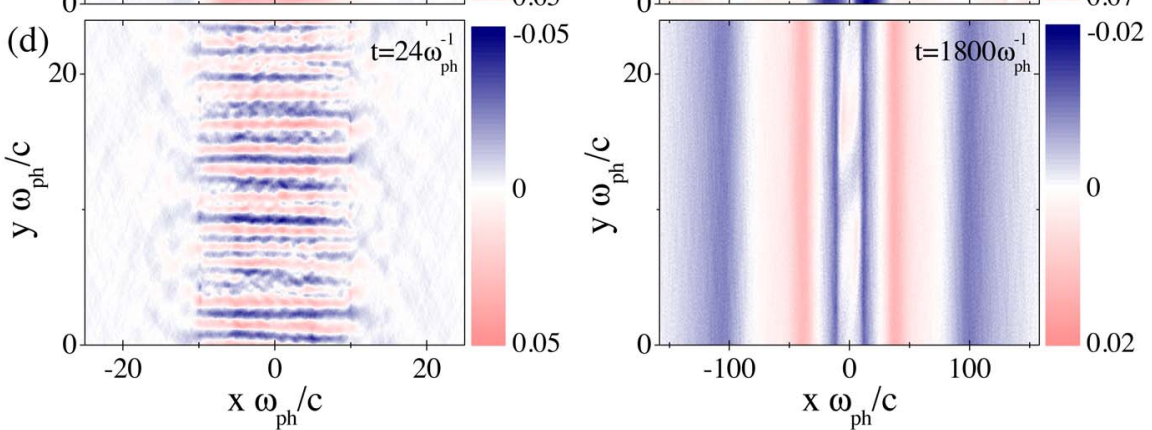

mode is now along the longitudinal axis (the new cold axis). However, because the plasma has been magnetized and because the anisotropy is weak, the magnetic field is also composed of modes with $\theta \neq 0$ (see Fig. 5). In other words, the wave vectors are not all purely longitudinal. Figure 9(a) indicates that for $t \gtrsim 2700 \omega_{p h}^{-1}$, the magnetic field is sufficiently intense to keep $p_{\perp}^{2}(t)=p_{\|}^{2}(t)$. As a result, the energy gained by ions is taken equally from the electron thermal energies in both directions [16].

To study the influence of a cold population, we ran a simulation with the same initial hot mean-square momentum as in Fig. 9(a) but with $\alpha=0.1$. Figure 9(b) shows that in this case, the magnetic energy grows much more slowly. According to Eq. (33) we expect $\Gamma^{m}=0.01 \omega_{p h}^{-1}$. This value is consistent with Fig. 9(b) but only for $t \lesssim 50 \omega_{p h}^{-1}$. As the anisotropy decreases, the growth rate $\Gamma_{P I C}^{m}$ at latter time is actually much lower. Figure 10(b) shows that the maximum magnetic field, obtained at $t \approx 600 \omega_{b h}^{-1}$, hardly emerges from the noise level. As a consequence $p_{\perp}^{2}$ is almost steady during the first stage of the expansion. Note that $p_{\|}^{2}$ is lower in Fig. 9(b) than in
Fig. 9(a) because $90 \%$ of the electrons are cold.

As electrons give progressively their longitudinal momentum to ions, $p_{\|}^{2}$ eventually becomes smaller than $p_{\perp}^{2}$ at $t$ $\approx 1200 \omega_{p h}^{-1}$. As in the case without cold electrons, an instability can thus develop again. The right column of Fig. 10(b) shows, however, that in contrast with Fig. 10(a), the wave vectors are almost perfectly longitudinal during this second stage. This is probably because the plasma is much less magnetized when the field restarts to grow. Despite this difference, Fig. 9(b) reveals that the expansion is very similar with and without a cold population, once the instability has developed for a second time. Indeed, the expansion becomes isotropic and the mean magnetic energy normalized by hot electron is almost the same in the two cases. Thus, the presence of cold electrons modifies the early time of the expansion and the time at which the longitudinal and transverse energies become equal, but it does not perturb much the behavior of the plasma at long time.

(b) Relativistic case. We now turn to the relativistic regime by considering a plasma with a hot energy $E_{h 0}$ 
$=1 \mathrm{MeV}$. Figure 9(c) shows that there is not much difference between the relativistic and nonrelativistic cases. In agreement with Fig. 6, the growth rate is just larger in the relativistic case (we measure $\Gamma_{P I C} \approx 0.41 \omega_{p h}$ ), so that the saturation of the magnetic field and the isotropization of the distribution function occur earlier. We remark also that, at maximum, about $1.2 \%$ of the initial hot energy has been converted to magnetic energy, while in Fig. 9(a) it was about $2 \%$. This is another consequence of the relativistic saturation observed in Fig. 6. Note that the absolute value of $E_{m}$ is, however, much larger in the relativistic case.

The left column of Fig. 10(c) shows that the most unstable wave number is quite the same as in Fig. 10(a). This is at first confusing since, according to Eq. (23), $k^{m}$ is expected to increase with $A$. But Fig. 9(c) shows that the anisotropy is already strongly reduced when $E_{m}$ is maximal, so that $A$ is almost identical at this time in Figs. 9(a) and 9(c), which explains why $k_{P I C}^{m}$ is also the same in both cases. During the second stage of the expansion, all the wave vectors lie in the longitudinal axis, while in (a) some modes had a transverse component. This suggests that modes with $\theta>0$ are more stable in the relativistic regime.

According to Sec. II B, the presence of a cold population with $\alpha=0.1$ is not expected to affect significantly the expansion when $A$ is large. For $A=v_{\| 0}^{2} / v_{\perp 0}^{2}-1 \approx 240$ [case of Fig. 9 (c)], the classical dispersion relation [Eq. (32)] suggests that the maximum growth rate should be divided by 1.3 for $\alpha$ $=0.1$. One-dimensional relativistic simulations with fixed ions and $\boldsymbol{k}=k_{y} \boldsymbol{e}_{\boldsymbol{y}}$ demonstrate that this result is not modified when relativistic effects are taken into account. Yet, we measure in Fig. 9(d) that the growth rate $\Gamma_{P I C}=0.17 \omega_{p h}$ is 2.4 smaller for $\alpha=0.1$ than for $\alpha=1$. This figure shows also that the mean magnetic energy by hot electron is more than 20 times lower in presence of cold electrons (instead of 9 in 1D simulations). Figure 10(d) further confirms that the addition of a cold population reduces the amplitude of the magnetic field and shows that it increases the wave number of the most unstable mode. This last point is also observed in 1D simulations and, in a lesser extent, in classical calculations [using Eq. (32) we find that $k^{m}$ should be multiplied by 1.7].

The differences between 1D and 2D simulations indicate that the plasma expansion influences the development of the Weibel instability. Indeed, as hot electrons transfer their momentum both to ions and cold electrons, via kinetic effects [31], the anisotropy decreases which hinders the growth of the instability. In (a) and (c) this cooling is quite small during the first stage, so that the effect of the expansion on the Weibel instability is weak. The stronger influence observed in (d) might be due to a significant transfer of momentum from hot electrons to cold electrons.

Despite a larger impact of the electron cooling on the development of the Weibel instability, the expansion is similar in (c) and (d), with two differences. First, because the amplitude of the magnetic field is smaller, the isotropization of the distribution function occurs later in (d) than in (c). Second, more kinetic energy is converted in magnetic energy during the second stage of the expansion than during the first, for the influence of cold electrons is much weaker during this second stage.

Figure 10(d) shows that, after the second development of the instability, the magnetic field is very weak in the center of the plasma. This is because this part of the plasma is stabilized by cold electrons, while the outer plasma contains only hot electrons. A similar effect is observed, although less clearly, in the absence of a cold population. In this case, it is due to the fact that the anisotropy is weaker in the unperturbed part of the plasma because of kinetic effects $[31,32]$, so that the magnetic field develops mainly in the expanding part of the plasma [16].

In conclusion, this numerical study demonstrates that the classical linear model presented in Sec. II is sufficient to understand most of the features of the expansion, and to predict the influence of various parameters. It is however inadequate to obtain precise values in the relativistic regime. As shown in Sec. II B, the addition of a cold population tends to stabilize the plasma and Fig. 9(d) suggests that this effect is stronger when hot electrons are relativistic. The influence of a cold population is much weaker during the second growth of the instability. As a result, the amplitude of the magnetic field, in the presence of cold electrons, is generally larger during the second stage of the expansion. In the next section we show that these results can be used to analyze the development of the Weibel instability during the interaction of an intense laser pulse with a thin foil.

\section{B. Laser heated plasma}

\section{Numerical conditions}

To complete this study, we consider now a plasma slab with an isotropic electron temperature of $500 \mathrm{eV}$, and we use a $2 \mathrm{D} 3 \mathrm{~V}$ relativistic and collisional PIC code to study the development of magnetic fields during the interaction of this plasma with an intense laser. The laser is a Gaussian pulse with a full width at half maximum of $\sigma=4.8 \lambda$, a half amplitude duration of $\tau=95 T$, and a full duration of $190 T$, where $\lambda=1 \mu \mathrm{m}$ is the laser wavelength and $T=c / \lambda$ is the optical period. This laser impinges on the plasma at normal incidence and reaches a peak intensity $I \lambda^{2}=1.2$ $\times 10^{19} \mathrm{~W} \mathrm{~cm}^{-2} \mu \mathrm{m}^{2}$. The plasma is a slab of initial width $L=2 \lambda$ and of electron density $n_{e 0}=100 n_{c}$, where $n_{c}$ $=m_{e} \omega_{0}^{2} \epsilon_{0} / e^{2}$ is the critical density.

The mesh size is $\Delta x=0.015 \mathrm{c} / \omega_{0}$ and the time step is $\Delta t$ $=0.0145 \omega_{0}^{-1}$, with $\omega_{0}$ as the laser angular frequency. The simulation box size is $240 \mathrm{c} / \omega_{0} \times 576 \mathrm{c} / \omega_{0}$ and there are 20 particles in each cell. Particles are reinjected with thermal velocities when they reach the transverse boundaries and are reflected when they reach the longitudinal boundaries. Electron-electron and electron-ion collisions are treated using the Monte Carlo method proposed by Nanbu [33]. For simplicity, the Coulomb logarithm is fixed to 12 , which corresponds to an initial mean collision frequency of about $0.3 \omega_{0}$. Note that this choice results in an overestimate of the collision frequency for cold electrons.

\section{Simulation results}

During the interaction of an overdense plasma with an intense laser pulse at normal incidence, electrons in the skin depth are accelerated in the longitudinal direction by the $\boldsymbol{v}$ $\times \boldsymbol{B}$ force [22]. A hot anisotropic population of electron is 


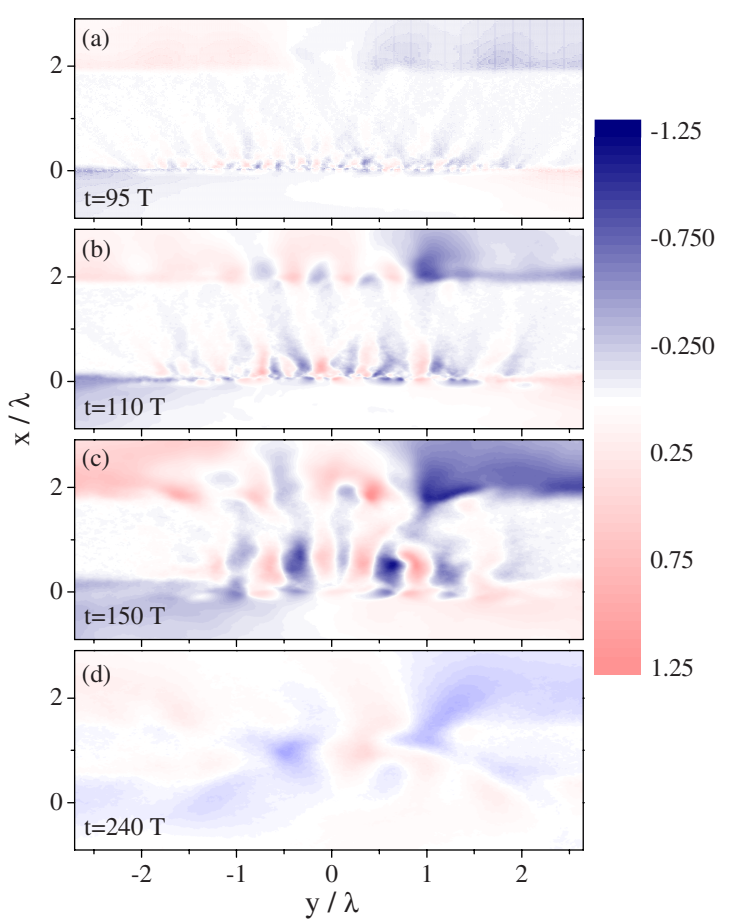

FIG. 11. (Color online) Magnetic field $B_{z}(x, y) /\left(m_{e} \omega_{0} / e\right)$ at different times, during the interaction of an intense laser pulse with a plasma slab. The laser comes from the $x<0$ half-space. The maximum of the pulse reaches the surface $x=0$, where the laser is reflected, at $t=120 T$. Its focal spot is centered at $y=0$.

thus created within a few laser optical cycles. In light of Sec. III A, this anisotropy is expected to lead to the growth of the Weibel instability.

Figure 11(a) shows that at $t=95 T$, magnetic fields have actually developed around the plasma surfaces. In particular, we notice the presence of intense fields in the expanding parts of the plasma ( $x \lesssim 0$ and $x \gtrsim 2 \lambda)$. They are however not due to the Weibel instability but to the limited size of the laser focal spot which induces temperature and density gradients [34]. In the following, we ignore these fields and we rather concentrate on the ones which are developing because of the Weibel instability in the inner plasma $(0 \leqq x \leqq 2 \lambda)$. These fields, which can be easily identified due to their large wave numbers, start to grow at $t \approx 60 T$, the time at which the laser amplitude at the plasma surface has reached $30 \%$ of its maximum value. As the anisotropy and the hot electron velocity at this time are, respectively, $A \approx 7.7$ and $v_{\|} \approx 0.16 c$, we expect from the linear theory a growth rate of $\Gamma^{m}$ $=0.6 \omega_{0}$. This is consistent with the simulation where the growth rate at $t \approx 60 T$ is $\Gamma_{P I C}>0.5 \omega_{0}$ (the precision is limited by the time resolution of the diagnostic). A good agreement between the theory and the simulation is also observed at $t=95 T$. At this time, the anisotropy has been reduced by the magnetic field $(A=2.2)$, so that the theoretical wave vector is $k^{m}=7 \omega_{0} / c$, which corresponds precisely to the numerical value evaluated from Fig. 11(a). This value then decreases in time as the anisotropy is further lowered [Figs. 11(b) and 11(c)].

Interestingly, we remark in Fig. 11(b) that the magnetic field, which almost vanishes in the center of the plasma, is quite intense at the rear side of the target (at $x \gtrsim 2 \lambda$ ). This is due to the presence of cold electrons in the plasma. According to Sec. II B a population of cold electrons actually "stabilizes" the plasma when the fraction of hot electrons $\alpha$ is much smaller than $1 /(A+1)$. At $t=60 T$ when the instability starts to develop, the fraction of hot electrons in the plasma bulk is about $\alpha \approx 0.15 \%$. For $A=7.7$, the growth rate is thus according to Eq. (33) $2.6 \times 10^{4}$ lower than in absence of cold electron. This prevents the instability to develop in this part of the plasma. The situation is quite different around the plasma-vacuum interfaces where for different reasons there is no cold population. At the front surface, the cold population has in fact been strongly heated by the laser, while at the rear surface, the magnetic field grows in an area which was initially in vacuum and which contains therefore no cold electron. Looking at Figs. 11(a)-11(c) we see that the magnetized area of the plasma increases in time. This is because the cold electron temperature near the magnetic front progressively increases, mainly due to resistive heating, so that the instability can develop in a larger part of the plasma.

The amplitude of the magnetic field is maximum around $t=150 T$, which corresponds to the time at which the laser amplitude at the plasma surface has dropped to $\approx 80 \%$ of its peak value. Figure 11(c) shows that there is still a thin layer of the plasma which is weakly magnetized at this time. Then, the amplitude of the magnetic field slowly decreases as the plasma expands (similarly to Fig. 9). This is illustrated in Fig. 11(d) which shows that at $t=240 T$ the magnetic field amplitude has been reduced by $\approx 3$.

Thus, the physics is very similar to the one analyzed in Sec. III A, except that in the present case the plasma conditions vary in space, and that during most of the laser-plasma interaction the laser is a source of anisotropy which feeds the instability. Note that we obtained very similar results using a 2D3V collisionless code which indicates that the influence of Coulomb collisions is weak during the time of the simulation for the chosen parameters. The magnetic field penetrates only slightly faster in the inner plasma without collisions.

\section{CONCLUSION}

This paper shows that, in an anisotropically heated plasma, magnetic fields in the megagauss range grow during the plasma expansion in two stages. During the first stage, the instability develops with wave vectors perpendicular to the expansion direction, while during the second one they are mainly parallel to this direction. In both cases, the development of the instability was analyzed using simple linear models. For classical electron temperatures, these models provide a quantitative description of the instability. In the relativistic regime, they do not lead to quantitative predictions, but can be used to estimate, for a given set of parameters, the significance of the Weibel instability and to assess the influence of a particular parameter.

The influence of a cold population has been studied in detail. In particular, we demonstrated that when the inverse of the hot electron fraction is larger than the anisotropy, the plasma is stabilized by the cold population. Thus, the instability can be inhibited during the first stage of the expansion. 
In agreement with Ref. [16], we showed that the influence of cold electrons is much weaker during the second stage of the expansion, so that intense magnetic fields eventually develop in all cases.

This last point is only true if the influence of Coulomb collisions is still weak when the anisotropy restarts to increase; otherwise, collisions will isotropize the plasma and prevent the instability to develop. According to Ref. [15] collisional effects become predominant when the mean collision frequency $\nu_{0} \gtrsim 100 c_{s 0} / L$, where $c_{s 0}=\sqrt{Z k_{B} T_{e} / m_{i}}$ is the ion acoustic velocity, that is, when $\left(n_{e} / 10^{21} \mathrm{~cm}^{2}\right)(L / \mu \mathrm{m}) /\left(T_{e} / \mathrm{eV}\right)^{2} \gtrsim 10^{-2}$. At the beginning of the second stage, the electron temperature is on the order of or higher than the initial temperature in the cold direction; we can thus assume $T_{e} \gtrsim 500 \mathrm{eV}$. Doing so we find that, for $L=1 \mu \mathrm{m}$, collisions will inhibit the second growth of the instability only if $n_{e} \gtrsim 2.5 \times 10^{24} \mathrm{~cm}^{-2}$. Obtaining such a high density after the first stage of the expansion is highly unlikely. This shows that collisions cannot play a significant role during the laser-plasma ion acceleration. For very cold initial electron temperatures they could, however, contribute to hinder the development of the instability in the inner part of the plasma.

Since the instability develops in a few femtoseconds $(\approx 0.4$ fs in the case of Fig. 2 ), the magnetic field can influence strongly the laser-plasma interaction and the plasma expansion. Its most direct impact is of course to isotropize the electron distribution function, which leads to a slower decrease of the electron temperature. In the classical limit, for instance, the temperature for $t \gg c_{s 0} / L$ decreases as $T_{e}$ $\propto t^{-2 / 3}$ with isotropization, and as $T_{e} \propto t^{-2}$ without it $[15,16]$. More importantly, the magnetic field influences the transverse width of the electron clouds at the plasma surfaces, and hence the emittance of accelerated ion beams $[17,18,35]$. As mentioned above, $\boldsymbol{B}$ may also perturb the coupling between the laser and the plasma. The amplitude of the self-generated magnetic field is actually of the same order of the one of the laser field $\left(1.2 m_{e} \omega_{0} / e\right.$ versus $3 m_{e} \omega_{0} / e$ at maximum in the case of Sec. III B). As this magnetic field varies in both time and space, it could perturb both temporally and spatially the plasma dynamics and thus influence the properties of energetic electron beams [36] and of high-order harmonics $[37,38]$ produced on dense plasmas. Lastly, it could also influence the propagation of electromagnetic waves (e.g., highorder harmonics) through the plasma [39].

\section{ACKNOWLEDGMENTS}

This work was performed using HPC resources from GENCI-IDRIS (Grant No. 2009-056044). C.T. acknowledges support of the AXA Research Fund and of Agence Nationale de la Recherche (Project No. ANR-06-BLAN0392). T.M.A. acknowledges support of the U.S. Department of Energy and of the Triangle de la Physique.
[1] F. N. Beg, A. R. Bell, A. E. Dangor, C. N. Danson, A. P. Fews, M. E. Glinsky, B. A. Hammel, P. Lee, P. A. Norreys, and M. Tatarakis, Phys. Plasmas 4, 447 (1997).

[2] R. A. Snavely et al., Phys. Rev. Lett. 85, 2945 (2000).

[3] E. L. Clark et al., Phys. Rev. Lett. 84, 670 (2000).

[4] A. Maksimchuk, S. Gu, K. Flippo, D. Umstadter, and V. Y. Bychenkov, Phys. Rev. Lett. 84, 4108 (2000).

[5] J. Fuchs et al., Nat. Phys. 2, 48 (2006).

[6] H. Schwoerer, S. Pfotenhauer, O. Jäckel, K. Amthor, B. Liesfeld, W. Ziegler, R. Sauerbrey, K. W. D. Ledingham, and T. Esirkepov, Nature (London) 439, 445 (2006).

[7] B. M. Hegelich, B. J. Albright, J. Cobble, K. Flippo, S. Letzring, M. Paffett, H. Ruhl, J. Schreiber, R. K. Schulze, and J. C. Fernández, Nature (London) 439, 441 (2006).

[8] L. Robson et al., Nat. Phys. 3, 58 (2007).

[9] T. Ceccotti, A. Lévy, H. Popescu, F. Réau, P. D’Oliveira, P. Monot, J. P. Geindre, E. Lefebvre, and P. Martin, Phys. Rev. Lett. 99, 185002 (2007).

[10] E. Lefebvre et al., New J. Phys. 12, 045017 (2010).

[11] A. V. Gurevich, L. V. Pariı̌skaya, and L. P. Pitaevskiǔ, Zh. Eksp. Teor. Fiz. 49, 647 (1965).

[12] J. E. Crow, P. L. Auer, and J. E. Allen, J. Plasma Phys. 14, 65 (1975).

[13] P. Mora, Phys. Rev. Lett. 90, 185002 (2003).

[14] P. Mora, Phys. Rev. E 72, 056401 (2005).

[15] C. Thaury, P. Mora, J. C. Adam, and A. Héron, Phys. Plasmas 16, 093104 (2009).

[16] C. Thaury, P. Mora, A. Héron, and J. C. Adam, Phys. Rev. E
82, 016408 (2010).

[17] A. J. Kemp, J. Fuchs, Y. Sentoku, V. Sotnikov, M. Bakeman, P. Antici, and T. E. Cowan, Phys. Rev. E 75, 056401 (2007).

[18] J. C. Adam, A. Héron, and G. Laval, Phys. Rev. Lett. 97, 205006 (2006).

[19] E. S. Weibel, Phys. Rev. Lett. 2, 83 (1959).

[20] D. W. Forslund, J. M. Kindel, K. Lee, E. L. Lindman, and R. L. Morse, Phys. Rev. A 11, 679 (1975).

[21] F. Brunel, Phys. Rev. Lett. 59, 52 (1987).

[22] W. L. Kruer and K. Estabrook, Phys. Fluids 28, 430 (1985).

[23] A. Bret, M.-C. Firpo, and C. Deutsch, Phys. Rev. E 70, 046401 (2004).

[24] B. D. Fried and S. D. Conte, The Plasma Dispersion Function (Academic, New York, 1961).

[25] J. D. Huba, NRL Plasma Formulary (Naval Research Laboratory, Washington,D.C., 2009), revised.

[26] U. Schaefer-Rolffs, I. Lerche, and R. Schlickeiser, Phys. Plasmas 13, 012107 (2006)

[27] P. H. Yoon, Phys. Plasmas 14, 024504 (2007).

[28] U. Schaefer-Rolffs and R. C. Tautz, Phys. Plasmas 15, 062105 (2008).

[29] R. L. Morse and C. W. Nielson, Phys. Fluids 14, 830 (1971).

[30] A. Stockem, M. E. Dieckmann, and R. Schlickeiser, Plasma Phys. Controlled Fusion 51, 075014 (2009).

[31] T. Grismayer, P. Mora, J. C. Adam, and A. Héron, Phys. Rev. E 77, 066407 (2008).

[32] P. Mora and T. Grismayer, Phys. Rev. Lett. 102, 145001 (2009). 
[33] K. Nanbu, Phys. Rev. E 55, 4642 (1997).

[34] K. A. Brueckner and S. Jorna, Rev. Mod. Phys. 46, 325 (1974).

[35] X. H. Yuan et al., New J. Phys. 12, 063018 (2010).

[36] G. Malka and J. L. Miquel, Phys. Rev. Lett. 77, 75 (1996).
[37] C. Thaury et al., Nat. Phys. 3, 424 (2007).

[38] C. Thaury, H. George, F. Quéré, R. Loch, J.-P. Geindre, P. Monot, and P. Martin, Nat. Phys. 4, 631 (2008).

[39] H. George, F. Quéré, C. Thaury, G. Bonnaud, and P. Martin, New J. Phys. 11, 113028 (2009). 